\title{
INVESTIMENTO SOCIAL PRIVADO ALÉM DOS ASPECTOS ECONOMICOS: COMPARTILHAMENTO DE CONHECIMENTO NA RELACÃO EMPRESA-ONGS
}

PRIVATE SOCIAL INVESTMENT BEYOND ECONOMIC ASPECTS: KNOWLEDGE SHARING IN THE RELATIONS BETWEEN COMPANIES AND NGOS

LA INVERSIÓN SOCIAL PRIVADA MÁS ALLÁ DE LOS ASPECTOS ECONÓMICOS: EL COMPARTIR CONOCIMENTOS EN LA RELACIÓN EMPRESA-ONGS

\author{
Mariana Galvão Lyra \\ Mestre \\ Pontifícia Universidade Católica de Minas Gerais \\ mglyra@gmail.com \\ Helio Zanquetto Filho \\ Doutor \\ Universidade Federal do Espírito Santo \\ zanquetto@gmail.com \\ Ricardo Corrêa Gomes \\ Doutor \\ Universidade de Brasília \\ rgomes@unb.br \\ Miriam de Magdala Pinto \\ Doutora \\ Universidade Federal do Espírito Santo \\ miriammagdalapinto@gmail.com \\ Submetido em: 03/10/2011 \\ Aprovado em: 08/03/2013
}

RESUMO

O conhecimento como moeda de troca nas relações entre organizações é um tema que vem crescendo na literatura nacional e internacional. O presente estudo teve como objetivo analisar algumas das relações interorganizacionais da Petrobras, com lócus exclusivo na UN-ES, buscando identificar e compreender aspectos que possam facilitar o compartilhamento de conhecimento entre os atores e alavancar a aprendizagem organizacional. Especificamente, foram selecionadas as relações interorganizacionais com ONGs que já participaram do Programa Ciranda Capixaba. Desta forma, foram realizadas quinze entrevistas com gestores da Petrobras e das ONGs para a coleta dos dados. A partir das entrevistas, fez-se sua transcrição para análise, que utilizou os elementos da análise de conteúdo. Como resultado, observa-se que tanto a Petrobras UN-ES quanto as ONGs buscam, com diferentes objetivos, legitimidade no processo de relacionamento. A adequação de linguagem se constitui como um dos elementos fundamentais para que o compartilhamento de conhecimento seja facilitado, sendo observado que as ONGs adaptam sua linguagem àquelas utilizadas pela Petrobras UN-ES, prevalecendo assim a lógica de haver um depositário da informação e, em contrapartida, um dominador das linguagens. Para superar as dificuldades gerenciais, criou-se a figura da "terceira pessoa ou organização", que funciona como intermediador da confiança necessária para melhorar a troca de informações. A pesquisa revelou o desejo entre as partes para colaborarem, de forma a criar mecanismos coordenados. Assim, essa pré-disposição para melhorar, 
apoiada na possibilidade de ganhos com a legitimidade por ambas as partes, faz com que seja possível haver benefícios acima do normal (rents) gerados em conjunto, uma vez que estes não podem ser gerados por uma das duas organizações de maneira isolada. Há uma necessidade de se refletir para além dos aspectos econômicos nas relações interorganizacionais com foco nos projetos socioambientais. Ou seja, pode-se avançar nas pesquisas, a fim de desenvolver conceitos centrais e as novas tecnologias de gestão dos relacionamentos interorganizacionais com foco na melhoria da gestão do conhecimento com organizações dessas características.

PALAVRAS-CHAVES: Compartilhamento de Conhecimento. Responsabilidade Social Corporativa. Relação Interorganizacional.

\section{ABSTRACT}

The idea of knowledge as a currency of exchange in interorganizational relations is growing in the international and national literature. This study analyzes some of the interorganizational relations of Petrobras, as an exclusive locus in the UN-ES, seeking to identify and understand aspects that could facilitate a sharing of knowledge between the actors, and promote organizational learning. Specifically, interorganizational relationships were selected with NGOs that had been involved in the Ciranda Capixaba Program. Fifteen interviews were conducted with managers of Petrobras and the NGOs for the data collection. The interviews were transcribed for subsequent analysis, using elements of content analysis. The results showed that both Petrobras and the NGOs are searching for legitimacy in their relationship processes, albeit with different aims. The adaptation of language is one of the fundamental elements for enabling knowledge sharing. It was noted that NGOs adapted their language to that used by Petrobras, with a prevalent logic that there is an information depository, and on the other hand, a dominator of languages. To overcome the management difficulties, the figure of "third person or organization" was created, which functions as an intermediary of the confidence needed to improve the exchange of information. The research showed the desire of the parties to collaborate, seeking to create coordinated mechanisms. This willingness to improve, supported by the possibility of legitimate benefits on both sides, makes it possible to gain kind of upper level benefits (rents) which are jointly generated, as they cannot be generated by either party on its own.

KEYWORDS: Knowledge sharing. Corporate Social Responsibility. NGO-business relations.

\section{RESUMEN}

El conocimiento como elemento de intercambio en las relaciones entre organizaciones es un tema que viene creciendo en la literatura nacional e internacional. El presente estudio tuvo por objetivo analizar algunas de las relaciones interorganizacionales de la Petrobras, con lócus exclusivo en la UN-ES, buscando identificar y comprender aspectos que puedan facilitar el compartir conocimientos entre los actores e impulsar el aprendizaje organizacional. Específicamente, fueron seleccionadas las relaciones interorganizacionales con ONGs que ya habían participado en el Programa Ciranda Capixaba. De esta manera, se realizaron quince entrevistas con gestores de la Petrobras y de las ONGs para la recolección de los datos. A partir de las entrevistas se llevó a cabo su transcripción para análisis, que utilizó los elementos del análisis de contenido. Como resultado se observa que tanto la Petrobras UN-ES como las ONGs buscan, con diferentes objetivos, legitimidad en el proceso de relacionamiento. La adecuación del lenguaje constituye uno de los elementos fundamentales para facilitar la participación en el conocimiento. Se observó que las ONGs adaptan su lenguaje al utilizado por la Petrobras UN-ES, prevaleciendo así la lógica de que existe un depositario de la información y, en contrapartida, un dominador de los lenguajes. Para superar las dificultades gerenciales se creó la figura de la "tercera persona u organización", que funciona como 
intermediador de la confianza necesaria para mejorar el intercambio de informaciones. La investigación reveló el deseo entre las partes de colaborar, creando mecanismos coordinados. Así, esa predisposición a mejorar, apoyada en la posibilidad de ganancia por ambas partes con la legitimidad, hace que sea posible que haya beneficios superiores a lo normal (rents) generados en conjunto, dado que estos no pueden ser generados por una de las dos organizaciones de manera aislada. Hay una necesidad de reflexionar, más allá de los aspectos económicos, sobre las relaciones interorganizacionales con foco en los proyectos socioambientales. Es decir que se puede avanzar en las investigaciones a fin de desarrollar conceptos centrales y las nuevas tecnologías de gestión de los relacionamientos interorganizacionales centrados en la mejoría de la gestión del conocimiento con organizaciones de esas características.

PALABRAS CLAVE: Compartir Conocimientos. Responsabilidad Social Corporativa. Relación Interorganizacional.

\section{INTRODUÇÃO}

O setor de Óleo e Gás nos cenários local, nacional e global tem realizado pesados investimentos nas áreas sociais e ambientais, sendo que essas não se constituem como sua atividade fim principal. Assim, compreender a contribuição desses investimentos para a gestão organizacional é um dos motivadores do presente estudo.

O engajamento com stakeholders é uma tentativa de descrever o comportamento de uma organização ao interagir com seu ambiente. Apoiada na teoria institucional, a legitimidade é alcançada se os padrões da prática organizacional estão em congruência com o amplo sistema social (SCOTT, 1987; POWELL; DIMAGGIO, 1991).

A interação da organização com o ambiente é analisada dentro dos estudos sobre relacionamento interorganizacional, sendo este o principal foco teórico deste estudo. Barringer e Harrison (2000) descrevem seis arcabouços teóricos mais utilizados para explicar a formação de relacionamento interorganizacional, incluindo custo de transação, dependência de recursos, escolha estratégica, teoria de stakeholder, aprendizagem organizacional e teoria institucional. Apesar de cada paradigma ser insuficiente para captar as complexidades da formação de relacionamento interorganizacional, o fato é que relações interorganizacionais podem ser justificadas por diversos aportes teóricos.

Alguns autores (CHILD, 1976; OLIVER, 1991; GREENING; GRAY, 1994; GOMES; LIDDLE; GOMES, 2010) defendem o uso da teoria de dependência de recursos e da teoria institucional como forma de embasar a relação entre uma empresa e os diversos atores que compõem seu ambiente.

Pesquisadores brasileiros (APA, 2008; LYRA, 2008; POSTAL; FONSECA, 2008) têm investigado a relação entre a empresa e o seu ambiente dentro da temática de responsabilidade social e sustentabilidade. Recentemente, dentro desta área, pesquisas internacionais foram desenvolvidas e vêm sendo encontradas recorrentemente ao longo dos anos na literatura: o estudo das parcerias firmadas entre empresas e organizações não voltadas para o lucro (AUSTIN, 2000; SELSKY; PARKER, 2005; HOLMES; MOIR, 2007; SEITANIDI, 2007).

Ancorado nos estudos de Dyer e Singh (1998), o presente estudo utiliza a relação interorganizacional como unidade de análise. Sendo assim, o foco desta pesquisa não está nos indivíduos, sequer nas organizações, mas sim nas relações existentes entre a Petrobras UN-ES e ONGs, ou seja, organizações que não possuam a mesma lógica de operação da empresa. Dentro destas relações, especificamente, pretende-se identificar o compartilhamento do conhecimento.

Volpon (2006, p. 145) desenvolveu um estudo com vários stakeholders da Petrobras, sendo um dos resultados da pesquisa que mais se aproxima do problema do presente estudo é o fato de desvendar uma fragilidade nos relacionamentos entre a Petrobras e ONGs consideradas como "fracas e com um viés oportunístico, o que limita o aproveitamento de recursos e a obtenção de resultados benéficos para ambas as partes".

Assim, o lócus do presente estudo foi a Relação Empresa - ONGs: O Programa Ciranda Capixaba, rede de projetos selecionados dentro da área de influência da Petrobras no Espírito Santo, que busca 
difundir suas experiências e metodologias, tendo como base a promoção da sustentabilidade das comunidades nas quais se inserem (PETROBRÁS, 2006).

O presente estudo atuou analisando algumas das relações interorganizacionais da Petrobras, com lócus exclusivo na UN-ES, buscando identificar e compreender aspectos que possam facilitar o compartilhamento de conhecimento entre os atores e alavancar a aprendizagem organizacional. Especificamente, foram selecionadas as relações interorganizacionais com ONGs que já participaram do Programa Ciranda Capixaba.

Para se atingir o objetivo geral, os seguintes objetivos específicos foram realizados:

- Analisar, preliminarmente, as relações da Petrobras UN-ES com os stakeholders envolvidos;

- Averiguar os tipos de conhecimento existentes nas partes; e

- Verificar o compartilhamento de conhecimento na direção bilateral (partindo e chegando de ambas as partes envolvidas na relação) ou na direção unilateral (uma parte deposita o conhecimento na outra parte da relação).

Pouca pesquisa tem sido devotada para a compreensão da gestão dos relacionamentos interorganizacionais. Para a evolução da literatura evitando a redundância, mais pesquisas deveriam ser direcionadas a este assunto. Outro ponto a se elucidar é que a maior parte dos relacionamentos interorganizacionais falha. Uma compreensão mais clara para as práticas e as técnicas de gestão que facilitam o sucesso contínuo desses relacionamentos é importante para direcionar a pesquisa e a prática (BARRINGER; HARRISON, 2000).

A visão relacional da firma de Dyer e Singh (1998) sugere que os recursos críticos de uma empresa devam ir além da fronteira dessa empresa e serem embebidos nas rotinas e nos processos interorganizacionais. A tese central da visão relacional é que duas ou mais organizações podem desenvolver relacionamentos que resultem em vantagem competitiva sustentável. A unidade de análise é o relacionamento interorganizacional. Ressalta-se que as organizações são compostas por pessoas, entretanto o presente estudo se limita à relação entre as organizações estudadas, não descendo até o nível pessoal de análise.

A pergunta que norteou o presente estudo, dentro do contexto dos relacionamentos interorganizacionais, é: como ocorre (e se ocorre) o compartilhamento de conhecimento na relação de organizações com ONGs?

A seguir, será apresentado o arcabouço teórico utilizado no presente estudo com o recorte do levantamento de estudos anteriores sobre as relações interorganizacionais e sobre o compartilhamento de conhecimento nessas interações. A metodologia fica a cargo de explicar como o estudo foi realizado, para então os resultados serem apresentados. As considerações finais ressaltam a contribuição teórica do presente estudo em ir além dos aspectos econômicos e observar os fluxos de conhecimento no relacionamento entre uma empresa e uma organização sem fins lucrativos.

\section{REFERENCIAL TEÓRICO}

\section{As Relações Interorganizacionais}

As relações interorganizacionais se referem a quaisquer tipos de contatos entre duas ou mais organizações, variando da forma concorrencial e antagônica para aquelas de natureza cooperativa, tanto entre organizações similares ou diferentes, envolvendo transações, fluxos e ligações de recursos (OLIVER, 1990). Para fins deste estudo, as definições de parceria, aliança, interação e colaboração serão consideradas como sinônimos de relações interorganizacionais.

A confiança é uma questão complexa e está no âmago da formação e do desenvolvimento dos relacionamentos. Contudo ela sozinha não consegue oferecer condições suficientes para se atingir os objetivos dessas parcerias. Assim, o desenvolvimento de relacionamentos interorganizacionais mais efetivos passa pelo "aprimoramento dos sistemas de gestão, envolvendo planejamento de longo prazo, confiança nas relações intra-organizacionais, mecanismos de controle e coordenação 
mais sofisticados, como também pela solidificação de relações sociais" (CUNHA; MELO, 2006 p. 20). Ainda, relacionamentos mais recentes possuem a confiança intermediada por uma terceira pessoa ou organização (CUNHA; MELO, 2006).

Barringer e Harrison (2000) analisaram seis arcabouços teóricos mais utilizados para explicar a formação de relacionamentos interorganizacionais, incluindo custo de transação, dependência de recursos, escolha estratégica, teoria de stakeholder, aprendizagem organizacional e teoria institucional (Figura 1). Apesar de cada arcabouço ser insuficiente para captar as complexidades da formação de relacionamento interorganizacional, o fato é que as relações interorganizacionais podem ser justificadas por diversos arcabouços.

Cada um dos seis arcabouços oferece uma perspectiva única na formação dos relacionamentos interorganizacionais. Consequentemente, pesquisadores podem se beneficiar ao considerar cada um dos arcabouços ao desenhar seus estudos. De toda forma, a mistura dos arcabouços teóricos pode prover um significado mais útil para compreender a formação das relações interorganizacionais. Existe um efeito 'halo' (julgamentos que possibilitem a interferência da parte no todo, contaminando o resultado) na pesquisa que diz respeito à formação de relacionamento interorganizacional (BARRINGER; HARRISON, 2000). O presente estudo, então, lança mão da combinação desses arcabouços.

Figura 1. Os arcabouços teóricos na formação dos relacionamentos interorganizacionais

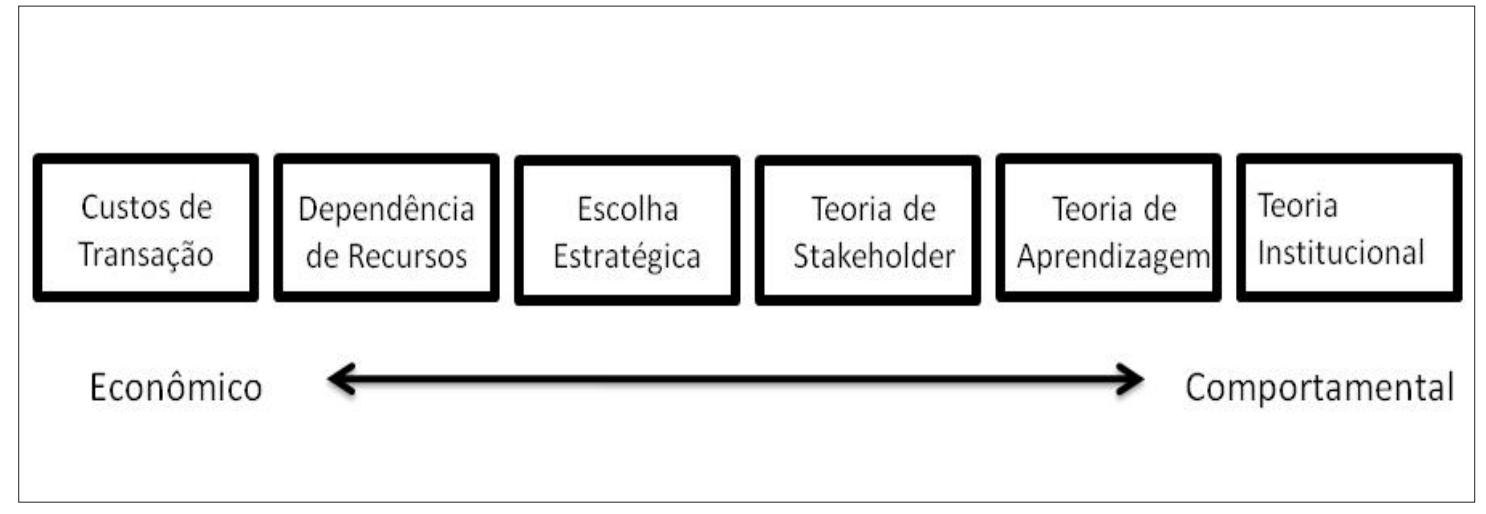

Fonte: Barringer e Harrison (2.000, p. 382) tradução livre.

Já que nenhuma das seis perspectivas é completa por si só, há uma necessidade de se considerar múltiplas perspectivas, na medida em que novas teorias são desenvolvidas e testadas. Outra consequência devido à falta de integração na literatura é que muitos termos diferentes são utilizados com significados aproximados. Por exemplo, existem muitos termos utilizados para identificar redes e o termo 'redes' é empregado para significar muitas coisas diferentes. Essa confusão de termos torna a disseminação efetiva de informação mais difícil e causa redundâncias nos esforços de pesquisa. Uma maior integração entre disciplinas pode reduzir estes problemas (BARRINGER; HARRISON, 2000).

Engajamento e parcerias com stakeholders são definidos como colaborações baseadas em confiança entre indivíduos e/ou instituições sociais com diferentes objetivos que só podem ser alcançados em conjunto (ANDRIOF; WADDOCK, 2002). São arranjos colaborativos ligando, muitas vezes, organizações de área dominadas por lógicas de operação (ex.: lucro, serviço social, vocacional) diferentes e muitas vezes conflitantes (DORADO; GILES; WELCH, 2009). A base para parcerias de sucesso é um acordo a respeito de regras para cooperação (ANDRIOF; WADDOCK, 2002).

Para Astley (1984), existe a negligência de uma variável particularmente importante no relacionamento organização-ambiente: a colaboração ou a ação conjunta entre organizações em questões de importância estratégica. Powell (1998), entretanto, chama a atenção para o fato de que a colaboração pode também se configurar como uma dimensão de competição, já que as empresas se voltam para o lado de fora em busca de uma variedade de recursos.

Existe uma tipologia específica de relacionamento interorganizacional que consiste em um arranjo colaborativo baseado em metas sinérgicas que ligam questões particulares que não poderiam ser 
realizadas de maneira isolada por cada uma das partes (CAPLAN, 2006). Os resultados esperados são novos produtos, serviços ou tecnologias, com menos custos de transação do que em alianças competidoras devido aos mecanismos de governança, que são mais efetivos (DYER; SINGH, 1998), e um desempenho de negócios superior ao que seria atingido se as organizações envolvidas decidissem colocar esforços individuais para alcançar os objetivos propostos da parceria (LAMBERT; EMMELHAINZ; GARDNER, 1996).

Considerando que os recursos críticos para a sobrevivência de uma organização por vezes se encontram fora dos seus limites, será por meio de relações com outras instituições, em rotinas e recursos interorganizacionais, que conseguirá obter o que deseja e oferecerá algo também a ser compartilhado. Nesta visão, os recursos não são simplesmente fortuitamente descobertos e explorados, mas produzidos pelas partes envolvidas na interação. A fonte desta criatividade e produção está nas ações organizacionais coletivas (ASTLEY, 1984). Assim, as vantagens de buscar este tipo de relacionamento incluem criação de ativos específicos da relação; rotinas de compartilhamento de conhecimento; complementaridade de recursos/capacidades e governança efetiva (DYER; SINGH, 1998).

A formação e a sustentabilidade das parcerias entre instituições com lógicas de operação diferentes foram analisadas em termos de direcionadores e ativadores com base nos estudos de Austin (2000). Direcionadores dizem respeito ao alinhamento de missão, estratégia e valores; às redes e aos relacionamentos pessoais; e à criação de valor. Os ativadores são: um claro propósito e atenção focada; expectativas mútuas; e processo de comunicação.

Um dos objetivos que une empresas e sociedade civil organizada (instituições sem fins lucrativos) é descobrir como desenvolver um relacionamento construtivo em conjunto. Esta busca é inerentemente normativa, já que busca explicar o que as empresas podem ou não fazer em prol do bem social, assim como demonstrar como obter legitimidade (ANDRIOF; WADDOCK, 2002).

\section{O compartilhamento de conhecimento}

O presente estudo, dentro do contexto dos relacionamentos interorganizacionais, busca identificar, nessas interações, como ocorre (e se ocorre) o compartilhamento de conhecimento. Para isso, inicialmente serão apresentadas algumas definições do conceito de conhecimento, o contexto dele na literatura, seus desdobramentos e conflitos, assim como estudos que tentaram criar um caminho para gerenciá-lo.

Wierzbicki (2007) defende a diversidade dos conceitos de conhecimento como um valor, dizendo que, embora o senso comum associe a ideia de conhecimento à herança e à experiência acumuladas por indivíduos (conhecimento individual) ou pela humanidade (conhecimento social), a engenharia do conhecimento atribui a este conceito uma relação muito mais direta com a informação, que é organizada e sintetizada por meio de modelos. Já Balestrin (2005) argumenta que, ao mesmo tempo em que essas correntes diferentes de definição geram discussões para o desenvolvimento da teoria do conhecimento organizacional, também suscitam ambiguidades e confusões tanto acadêmicas quanto empresariais.

Nonaka e Takeuchi (1997) têm sido citados recorrentemente nos estudos sobre conhecimento (COURTNEY, 2001; BALESTRIN, 2005; WIERZBICKI, 2007; JANOWICZ-PANJAITAN; NOORDERHAVEN, 2008; YUSUF, 2008; LUCCHI, 2009) e possuem foco na criação do conhecimento. Defendem dois tipos de conhecimento que se inter-relacionam: o tácito e o explícito. O tácito seria pautado na experiência pessoal do indivíduo, suas ações, crenças, valores e ideais. Já o conhecimento explícito é institucionalizado na forma física de manuais, ou arquivado em dispositivos, fazendo parte da memória organizacional, e podendo ser utilizado em atividades atuais e futuras.

O conhecimento na gestão contemporânea implica uma dificuldade imposta ao pragmatismo da gestão tradicional devido à sua forte dimensão tácita (BALESTRIN, 2005). Existe uma tendência ocidental de simplificar a natureza do conhecimento organizacional ao privilegiar a natureza explícita e individual em detrimento da natureza tácita e coletiva do conhecimento (COOK; BROWN, 1999).

A principal diferença entre o conhecimento na perspectiva intrapessoal e interorganizacional está na unidade de análise. Ao optar por analisar o presente estudo sob a ótica relacional, ou seja, 
das relações entre organizações, está-se ciente de que esta opção extingue aspectos inerentes ao indivíduo, como os psicológicos e sócio-históricos; e ao grupo, como a cultura organizacional presente em aglomerações de uma mesma instituição.

Sendo assim, Tonet e Paz (2004) defendem que, para ocorrer o compartilhamento de conhecimento, há a necessidade de existir uma linguagem comum entre as pessoas envolvidas no processo. Ocorre que, muitas vezes, o conhecimento a ser compartilhado envolve vocabulário inovador para os receptores, ou envolve termos que acabam sendo decodificados de forma diferente daquela pretendida pelo emissor. Pode ocorrer também que o próprio emissor tenha dificuldade em comunicar aquilo que deseja compartilhar.

O acúmulo de conhecimento por parceiros por meio de alianças pode ser de vários tipos. Parceiros adquirem conhecimentos que podem ser úteis nos relacionamentos colaborativos em geral, assim como o conhecimento de um parceiro específico pode ser de importância crucial para a forma com que a colaboração se desenvolve. Também relevante no contexto das alianças é o conhecimento substantivo que os parceiros utilizam no alcance de seus objetivos de negócios, como, por exemplo: conhecimento tecnológico, conhecimento gerencial e conhecimento de mercado (JANOWICZPANJAITAN; NOORDERHAVEN, 2008).

Esse conhecimento substantivo pode ter duas fontes: de um lado, parceiros podem aprender juntos no curso da colaboração e desenvolver conjuntamente novas capacidades e habilidades. Por outro, cada um dos parceiros pode levar tal conhecimento para a colaboração, assim dando ao outro uma oportunidade de acessar competências e habilidades desenvolvidas e adquiridas antes de entrarem na colaboração em foco (JANOWICZ-PANJAITAN; NOORDERHAVEN, 2008).

Dyer e Singh (1998) argumentam que organizações que colaboram podem gerar rents relacionais por meio de ativos específicos de relação, rotinas de compartilhamento de conhecimento, doação de recursos complementares e governança efetiva. A visão relacional tem identificado o mecanismo isolante que preserva os rents relacionais gerados por meio de colaborações interorganizacionais efetivas.

Rent relacional é definido como um benefício 'acima do normal' gerado em conjunto num relacionamento de troca que não pode ser gerado por nenhuma das organizações de maneira isolada e que só pode ser criado por meio de contribuições idiossincráticas de parceiros de alianças específicas. Em resumo, num nível fundamental, rents relacionais são possíveis quando os parceiros da aliança combinam, trocam ou investem em ativos idiossincráticos, conhecimento e capacidades/ recursos, e/ou empregam mecanismos efetivos de governança que diminuam os custos de transação ou permitam a realização de rent por meio de uma combinação sinérgica de ativos, conhecimento ou capacidades (DYER; SINGH, 1998).

Assim, parceiros da aliança podem gerar rents desenvolvendo rotinas de compartilhamento de conhecimento interorganizacionais superiores. Dyer e Singh (1998) definem uma rotina de compartilhamento de conhecimento interorganizacional como um padrão regular de interações interorganizacionais que permite transferência, recombinação, ou criação de conhecimento especializado. Essas interações são processos interorganizacionais institucionalizados que são propositalmente desenhados para facilitar as trocas de conhecimento entre parceiros de aliança.

Powell (1998) afirma que existem habilidades necessárias para se passar o conhecimento de um projeto para o outro e de uma unidade para outra. Sem essas habilidades, o conhecimento gerado não se perpetua e é perdido. Segundo o autor, na maioria das empresas existem pessoas-chave que funcionam como gestores de contatos, conselheiros de casamento e corretores honestos. Esses indivíduos fornecem a cola que sustenta os relacionamentos entre as partes que possuem amplas oportunidades para questionar suas intenções e interesses.

Uma dificuldade chave para relacionamentos intersetoriais é superar as diferentes formações e experiências profissionais e valores dos parceiros que podem tornar a troca de conhecimento especialmente difícil. Alianças com organizações não voltadas para o lucro podem proporcionar um desafio aos gestores que, por vezes, subestimam a complexidade de lidar com tais grupos. As organizações sem fins lucrativos possuem interesses e culturas diferentes que os gestores precisam avaliar com precisão, caso queiram que a parceria origine algum valor (HOLMES; MOIR, 2007).

Retomando a pergunta de pesquisa do presente estudo, dentro do contexto dos relacionamentos interorganizacionais, é como ocorre (e se ocorre) o compartilhamento de conhecimento na relação 
entre a Petrobras UN-ES e as ONGs do Programa Ciranda Capixaba. No próximo capítulo será apresentada a metodologia escolhida na tentativa de resolver o problema de pesquisa.

\section{PERCURSO METODOLÓGICO}

As relações empresa-ONG têm sido vistas como parcerias em que há um repasse financeiro por parte da empresa em troca do desenvolvimento de um projeto específico de cunho ambiental ou social. Este estudo analisou outros fluxos de troca que poderiam ocorrer nessas relações, utilizando para isso o conceito de compartilhamento de conhecimento.

A presente pesquisa configura-se como estudos de casos múltiplos. De acordo com Yin (2001, p. 33), "a pesquisa de estudo de caso pode incluir tanto estudos de caso único quanto estudos de casos múltiplos" e podem empregar múltiplos níveis de análise dentro de um mesmo estudo. Desta forma, estudos de casos são realizados na tentativa de descobrir e entender - por meio de uma investigação empírica - determinado fenômeno contemporâneo dentro de seu contexto da vida real, especialmente quando os limites entre o fenômeno e o contexto não estão claramente definidos (STRAUSS; CORBIN, 1990; YIN, 2001). Este estilo de pesquisa pode ser utilizado para estudar organizações, grupos e indivíduos. Para o presente estudo, foi utilizada a ótica relacional, ou seja, o foco de análise está nas relações entre organizações, e extingue aspectos inerentes ao indivíduo.

Em termos extremos de um continuum, os paradigmas são ou positivistas ou fenomenológicos. Poucos são os que atuam em suas formas puras.

O presente estudo está mais voltado para o paradigma fenomenológico, uma vez que considera a realidade como uma construção social, num processo indutivo de desenho emergente, em que as categorias são identificadas durante o processo de pesquisa. Como não tem a pretensão de atuar como uma "forma pura" de abordagem, possui também características presentes em estudos eminentemente positivistas, tais como estilo formal de escrita, na voz passiva.

Dentro do paradigma positivista, há uma etapa que consiste na elaboração de constructos, o que, por sua vez, é o processo de comparação constante entre os dados e o constructo de forma a acumular evidências das diversas fontes, construindo um único e bem definido constructo (EISENHARDT, 1989).

Considerando a análise das relações interorganizacionais entre uma empresa e seus stakeholders como fenômenos que não possuem seus limites claramente definidos com relação aos contextos que atuam, o presente estudo busca analisar a partir do relacionamento interorganizacional como unidade de análise (DYER; SINGH, 1998) como o compartilhamento de conhecimento ocorre nessas relações, a fim de contribuir para o progresso de futuras pesquisas neste campo.

Em contato direto dentro da Gerência do Programa Ciranda Capixaba, planilhas com informações acerca das ONGs já apoiadas ao longo dos ciclos do Programa foram encaminhadas para a pesquisadora, assim como um histórico do Programa. De posse deste material, foi constatado que a amostra era demasiadamente grande quando contrastada com os recursos de tempo e prazo do presente estudo: 64 ONGs localizadas ao longo do Estado do Espírito Santo.

Assim, inicialmente foram excluídas as ONGs que não faziam parte da grande Vitória, utilizando-se o critério de limitação geográfica. Como ainda assim o número inicial de ONGs era bastante elevado para um estudo aprofundado, dada as limitações de financiamento, tempo e deslocamento da pesquisadora, dentre as sete microrregiões que integram a Grande Vitória, foram escolhidas aquelas que possuíam o maior número de ONGs assistidas pelo Programa Ciranda Capixaba. Feita a primeira delimitação metodológica empírica, todas as 17 ONGs foram contatadas. Muitas das ONGs, mesmo tendo um espaço físico próprio, pediam o envio do questionário por e-mail ou realização da entrevista por telefone, visto que a maioria dos gestores possuem outros trabalhos (ou porque não se dedicam com exclusividade à ONG ou porque já não estão mais trabalhando nelas atualmente), às vezes fora do Estado do Espírito Santo. Estes pedidos foram respeitados.

Desta forma, ao longo da trajetória de tentativas de contato do universo de 17 ONGs, muitas delas não mais existiam, e em outras não se estabeleceu o contato mesmo depois de diversas 
tentativas. Como resultado, 9 ONGs responderam ao questionário semiestruturado e dessas foram realizadas entrevistas com apenas quatro instituições.

Ao todo foram realizadas 15 entrevistas. Quatro entrevistas foram feitas com representantes de ONGs, onze entrevistas foram realizadas com representantes da Petrobras (relação com ONGs). As entrevistas foram transcritas e sua análise de conteúdo foi feita a partir dessas transcrições realizadas (BARDIN, 1977). Deve-se destacar que não foram estabelecidas categorias "a priori" para análise. Além das informações coletadas por meio de entrevistas, documentos dos projetos, folders e algumas ferramentas de trabalho foram disponibilizadas para a presente pesquisa.

O processo de análise consistiu em buscar convergências entre os dados coletados no campo de pesquisa e os assuntos supracitados no referencial teórico no que diz respeito ao compartilhamento de conhecimento. Ao longo da análise, as categorias foram se formando na medida em que apareciam de maneira recorrente nos materiais de coleta e, assim, foram delineando os blocos que se configurariam como os resultados da pesquisa.

\section{RESULTADOS E DISCUSSÕES}

Nessa seção é apresentada a análise da pesquisa empírica com trechos das entrevistas reproduzidos, a fim de exemplificar melhor as questões levantadas. Algumas vezes eles foram reproduzidos com pequenas adaptações de forma a suprimir vícios de linguagem, sem prejuízo da mensagem e mesmo do estilo de comunicação. Tal procedimento foi necessário, pois a leitura de citações diretas sem estes pequenos ajustes ficaria cansativa e dificultaria - em alguns casos - a apreensão da mensagem do entrevistado. Cada item abaixo corresponde aos constructos utilizados como pano de fundo para a análise das entrevistas realizadas.

\section{Adequação de linguagem}

O vocabulário de gestão, necessário para o bom andamento dos projetos, era algo novo e diferente para a maioria das ONGs que pleiteavam os recursos da Petrobras. Muitas delas nunca haviam feito uma parceria com uma empresa (muito menos do porte da Petrobras) antes, e isto transpareceu fortemente a necessidade de alinhar e adequar a linguagem entre as partes para se estabelecer um contato de forma mais eficiente. Segue trecho da transcrição da fala em entrevista realizada com a comunicação da Petrobras (ECP) sobre esse tema:

(...) porque a grande dificuldade da gestão do projeto, que a gente via, é porque eles não entendiam a linguagem dos cursos e tal, então nós proporcionamos um intercâmbio. Esse intercâmbio fez com que eles criassem uma comunicação própria. (ECP02)

A primeira tentativa de solução desenvolvida pela equipe da Petrobras foi a de contratar consultores e cursos especializados em terceiro setor, porém, com a baixa adesão e assiduidade por parte das pessoas pertencentes às ONGs, principalmente dos seus gestores, a empresa decidiu reavaliar esta decisão. Assim, foi percebido que cursos não supriam a dificuldade das ONGs em compreender o vocabulário inovador da empresa, porque os condutores do processo não compreendiam a realidade e o vocabulário advindo dela por parte dos integrantes das ONGs.

Diante desta constatação, surgiu a oportunidade para a equipe da Petrobras pensar em proporcionar o contato entre as pessoas que trabalhavam nas diferentes ONGs que integravam o Programa Ciranda Capixaba. Esta alternativa se configurou como um cenário para trocarem experiências e conhecimentos, resolvendo a dificuldade de diferença de vocabulário por meio da seguinte dinâmica: existindo diferentes ONGs em diferentes níveis de desenvolvimento, ao colocar todas em um mesmo ambiente, automaticamente, com a intensificação da convivência e do relacionamento, os problemas, as soluções, as dificuldades e os desafios enfrentados por cada uma e todas elas, acabavam sendo semelhantes e, assim, juntos conseguiam desenvolver progressos no compreender e executar da gestão exigida pela parceria com a empresa.

Desta forma, a repetição das edições do Programa Ciranda Capixaba permitiu à equipe da Petrobras se adaptar e aprender com as ONGs. Atualmente a equipe da empresa afirma que conhece 
bem a linguagem das ONGs e se utiliza deste fato para facilitar o compartilhamento do conhecimento com seus gestores.

Assim, pode-se afirmar que houve um alinhamento sobre a dificuldade inerente em se compartilhar o conhecimento quando há necessidade prévia de adequação da linguagem, conforme defendem Tonet e Paz (2004).

\section{Presença de uma terceira parte no relacionamento}

A Petrobras contratou a ONG02 para facilitar a interação e o aprendizado conjunto das ONGs na adequação da linguagem para execução das tarefas de gestão exigida pela parceria junto ao Programa Ciranda Capixaba. Assim, essa ONG realizou, via financiamento do Programa Ciranda Capixaba, um trabalho um pouco diferente das demais ONGs, uma vez que desenvolveu atividades em prol do próprio programa. A percepção da Petrobras sobre essa iniciativa é apresentada na transcrição a seguir:

(...) Essa troca de experiência, esse intercâmbio que a gente proporcionou, fez eles [sic] melhorarem bastante na gestão. (...) [Pelo que estou entendendo, é necessário um intermediário para poder fazer essa troca?] É mais ou menos isso mesmo (...). E a gente acaba fazendo muito o meio de campo, mesmo [sic]. (ECP02)

Utilizar uma organização não voltada para o lucro para fomentar e incrementar discussões, ações e aprendizado para o grupo de ONGs participantes do programa diminuiu a distância de vocabulário existente e facilitou a adequação e o desenvolvimento das ONGs, a fim de garantir as exigências do programa. Segundo os entrevistados, a experiência foi válida, sendo possível perceber que a presença de uma terceira pessoa na parceria foi uma alternativa de sucesso que conseguiu intermediar os interesses da empresa com relação à adequação da linguagem.

\section{Diferentes formações e experiências profissionais dos parceiros}

Tanto na equipe da Petrobras quanto na composição dos participantes do Programa pela parte das ONGs, a formação e experiência profissional era muito diversa. Este ponto pode ser positivo e atuar incrementando processos e resultados, porém, em sua antítese pode dificultar o compartilhamento de conhecimento.

De acordo com o identificado, as ONGS se preocupam em aprender sobre gestão para poder atuar diretamente com as demandas da Petrobras para o desenvolvimento do projeto. $O$ fato de não terem muita experiência ou formação prévias nesta área não pareceu ter sido empecilho para o desenvolvimento posterior destas habilidades de modo a garantir o êxito do projeto.

Pelo lado da empresa, ressalta-se a importância de se formar internamente não só os profissionais da produção como também as pessoas que trabalham com a administração nas organizações do terceiro setor, com vias à colaboração que este tipo de conhecimento pode agregar no desenvolvimento dos projetos não só junto ao Programa Ciranda Capixaba, mas em outros relacionamentos com empresas e parceiros. Além disso, pode-se ver um relato da dificuldade causada pela falta de qualificação na área de gestão organizacional pelas ONGs.

\footnotetext{
É importante também pensar nisso quando for fazer o projeto. De qualificar as pessoas da comunidade ou mesmo, até, às vezes, contratar pessoas qualificadas para administrar o negócio que está aparecendo, mesmo que seja um negócio superficial, né. (...) Muitos projetos estão mais voltados para capacitar, por exemplo, o padeiro, capacitar a costureira, mas não (...) capacitar o administrador deste negócio que está aparecendo. (ECP03)
}

A assimetria na formação gerencial entre os gestores da Petrobras e das ONGs já era esperada e foi identificada. Entretanto esse fato, além de ser reconhecido pela Petrobras, não apresenta resistência por parte dos gestores das ONGs. Desta forma, foi identificado que o aprendizado gerencial dá-se de forma unilateral e é aceito por ambas as partes. Entretanto se deve destacar que, para avançarem em direção à melhoria dos processos gerenciais, é necessário que haja uma capacitação contínua dos gestores das ONGs de forma que essa assimetria possa ser reduzida no futuro, propiciando assim redução na assimetria da dependência na formação gerencial. 
O conhecimento substantivo do tipo gerencial foi trazido pela Petrobras e repassado para as ONGs via capacitação. Há um alinhamento entre os entrevistados da empresa e os das ONGs, criando, assim, uma indicação maior da validade desta informação.

Percebeu-se que as integrantes das ONGs consideram isto positivo, pois passam a incorporar este aprendizado no cotidiano de trabalho da organização e consideram o conhecimento adquirido como uma melhora em seus trabalhos.

O conhecimento substantivo de conhecimento gerencial foi também feito em conjunto pelas partes da parceria e não somente levado por uma das partes até a outra, por meio de um conhecimento prévio, anterior à parceria. As ONGs possuíam dificuldades e precisavam de uma adaptação nos termos de gestão da parceria com a empresa. Assim, a Petrobras identificou este problema e internamente buscou atender esta demanda das organizações para melhorar o seu relacionamento com elas. Esta ideia de mutualidade aparece, portanto, pela primeira vez nesta relação, indicando que, corroborando com Janowicz-Panjaitan e Noorderhaven (2008), existem duas fontes de conhecimento substantivo e, no caso do conhecimento gerencial para a relação ONGs-Petrobras, as duas formas foram encontradas.

Então, (...) não foi nem o que a gente aprendeu com eles, mas é a gente gostaria que... deste conhecimento que eles tem, a gente conseguisse levar pra outras organizações que tem dificuldades para se sustentar (...) eles têm uma facilidade, eles correm atrás para captar recursos. (ECP02)

Pode se depreender que a empresa não tinha o conhecimento substantivo de mercado de que era possível uma ONG ter seu quadro composto exclusivamente por voluntários e isto não atrapalhar o cotidiano da instituição. Este foi um conhecimento substantivo trazido pela ONG03 à parceria que, provavelmente, não será utilizado pela Petrobras, com a finalidade de atingir seus objetivos de negócio de modo geral. Entretanto se acredita que este aprendizado que a empresa obteve atue diretamente no seu relacionamento futuro com as outras ONGs, já que modifica a maneira de enxergar a gestão voluntária de instituições sem fins lucrativos.

[Na sua opinião, o que você acha que a Petrobras aprendeu com vocês?] Eu acho que aproxima mais a empresa da necessidade da população, principalmente de baixa renda, né [sic]. Eu acho que o principal é isso porque às vezes a gente que tá de fora não percebe a problemática (...) então, é muito complicado, né [sic]. E eu acredito que a maioria das pessoas não conseguem visualizar isso. Então eu acho que com esse projeto eles devem ter começado a ver as coisas de uma outra forma, né [sic]? Ver realmente como é esta realidade, da necessidade deste projeto. (EONG03)

Pode-se inferir que surgiu um tipo de conhecimento como resultado da pesquisa que não está presente na classificação dos três tipos de conhecimento propostos por Janowicz-Panjaitan e Noorderhaven (2008). Aparentemente este conhecimento não é gerencial, tecnológico ou de mercado, mas segundo as ONGs e a Petrobras foi possibilitado pela interação da empresa com as ONGs.

De Long e Fahey (2000) sugerem que existe um conhecimento amplamente tácito que só pode ser gerado em conjunto. Sua presença é refletida como uma habilidade de se colaborar efetivamente. Percebe-se que este ideal de cooperação foi encontrado nas respostas e que, portanto, consistem em uma classificação adicional aos conhecimentos tecnológicos, de mercado e gerencial propostos por Janowicz-Panjaitan e Noorderhaven (2008).

\section{Geração de rents}

A geração de rents foi o último aspecto a ser analisado por necessitar de análise da presença ou ausência dos quesitos analisados anteriormente.

Conforme destacam Dyer e Singh (1998), percebe-se que houve melhorias em ambas as partes a partir de resultados que foram alcançados em conjunto. Tanto a Petrobras quanto as ONGs percebem que existiram ganhos relacionais únicos e proporcionados pela troca entre eles durante o tempo de interação. No caso do relacionamento ONGs-Petrobras, apreende-se que o resultado 'acima do normal' foi possível não por um motivo isolado, mas pelo alinhamento de aspectos anteriores, como pode ser observado a seguir. 
Assim, pode-se afirmar que houve um alinhamento sobre a dificuldade inerente em se compartilhar o conhecimento quando há necessidade prévia de adequação da linguagem, conforme defendem Tonet e Paz (2004). Percebe-se a presença de um terceiro elemento não só para fins de garantias à confiança no relacionamento entre as partes da parceria, mas também como um instrumento efetivo para o gerenciamento dos relacionamentos e facilitação do compartilhamento de conhecimento, o que confirma as pesquisas de Powell (1998) e Cunha e Melo (2006). Dadas as diferentes formações e experiências profissionais dos parceiros, o aprendizado ainda está sendo unilateral, fato destacado como necessário para ser trabalhado por Holmes e Moir (2007). Analisando o conhecimento substantivo, verifica-se que este conhecimento não foi gerado em conjunto, mas levado pela Petrobras até às ONGs. Assim, seria melhor que cada um dos parceiros pudesse levar tal conhecimento para a colaboração, dando ao outro uma oportunidade de acessar competências e habilidades desenvolvidas e adquiridas antes de entrarem na colaboração em foco, como defendem Janowicz-Panjaitan e Noorderhaven (2008).

\section{CONSIDERAÇÕES FINAIS}

O presente estudo atuou analisando algumas das relações interorganizacionais da Petrobras, com lócus exclusivo na UN-ES, buscando identificar e compreender aspectos que possam facilitar o compartilhamento de conhecimento entre os atores e alavancar a aprendizagem organizacional. Desta forma, a partir da análise da tabulação das entrevistas foi possível averiguar aspectos relacionados ao compartilhamento do conhecimento, sendo que, para isto, foi necessário conhecer os motivadores, os atores envolvidos e a dinâmica do relacionamento.

Foi percebido que um dos direcionadores da parceria é a busca por resultados que vão além das fronteiras do relacionamento, ou seja, na legitimidade de outros stakeholders (acionistas, sociedade, fornecedores, mídia, por exemplo). Cabe observar que a legitimidade é um elemento importante tanto para a Petrobras, quanto para as ONGs. A presença da legitimidade no relacionamento ONGs-Petrobras pode atuar positivamente para as partes se motivarem, a fim de desenvolverem um relacionamento e, possivelmente, criarem melhores condições para o compartilhamento do conhecimento.

Uma ampliação do foco dos benefícios tácitos e explícitos com relação aos aprendizados que a Petrobras pode ter via compartilhamento de conhecimento com as ONGs poderia gerar rents para a empresa e tal possibilidade vem sendo subestimada por ela.

As ONGs apresentaram uma necessidade de conhecimento gerencial para gerir o relacionamento com a Petrobras. Esta necessidade corresponde à adequação da linguagem entre as partes, a qual foi necessária para o bom desenvolvimento da parceria. A Petrobras, por sua vez, atuou fornecendo cursos e capacitações na tentativa de minimizar este problema. Sendo assim, foram mais efetivos os cursos e as capacitações que não apresentavam necessidade de adaptação de linguagem entre o emissor e o receptor do conhecimento a ser transmitido.

Atuando também para facilitar o compartilhamento de conhecimento gerencial, a ONG02 atuou como a terceira pessoa/organização da relação ONGs-Petrobras. A presença de trainees nas ONGs participantes do Programa Ciranda Capixaba foi uma forma de facilitar o compartilhamento de conhecimento interorganizacional, mas, principalmente, pode-se inferir que, por meio desta iniciativa, este conhecimento foi sedimentado dentro das ONGs, expandindo, assim, o conhecimento nessas organizações, já que mesmo depois da saída do trainee acredita-se que o conhecimento continue presente na instituição, tácita ou explicitamente.

Foram encontradas indicações de que as ONGs possuíram dificuldades em compreender e se adequar à linguagem da Petrobras de gestão de suas parcerias. A categoria prestação de contas foi o maior aprendizado das ONGs tanto na opinião delas, quanto na opinião dos funcionários da Petrobras. Constata-se, ainda, com relação à adequação da linguagem, que foram as ONGs que se adaptaram às demandas da Petrobras. Verificou-se a existência de assimetria na formação gerencial, sendo que a Petrobras é reconhecida por ambos, como a mais bem-preparada. Adicionalmente, pode-se concluir que esta assimetria contribui fortemente para que a linguagem das ONGs seja adaptada para a linguagem Petrobras. De forma complementar, é importante não apenas a redução dessa assimetria, mas sim a capacitação das ONGs de forma que possam ser capazes de se capacitarem gerencialmente, sem depender do suporte da Petrobras. Desta forma, pode-se ter uma sustentabilidade na formação gerencial por parte destas. Esta é uma importante barreira a ser transposta. 
Foi verificada a presença de um tipo de conhecimento que flui a partir das ONGs para a Petrobras. Ele foi classificado como um conhecimento social que reflete a habilidade de cooperação conjunta entre os envolvidos no relacionamento. De fato, acredita-se que, por trás desta habilidade, esteja uma motivação maior, que pode residir no idealismo ou na solidariedade, visto que muitos dos que atuam no terceiro setor e/ou na área de responsabilidade social possuem estes valores. Estas características representam, portanto, um desejo de contribuir e cooperar independente dos objetivos profissionais atrelados às funções que desempenham em suas organizações, algo como uma qualificação extra que atua incrementando positivamente o ambiente para a construção e o compartilhamento do conhecimento no relacionamento.

Os autores da presente pesquisa consideram que a contribuição desta dá-se pela necessidade de se refletir para além dos aspectos econômicos nas relações interorganizacionais com foco nos projetos socioambientais. Ou seja, como se pode avançar nas pesquisas, a fim de desenvolver conceitos centrais e as novas tecnologias de gestão dos relacionamentos interorganizacionais com foco na melhoria da gestão do conhecimento com organizações dessas características. Um dos pressupostos da relação interorganizacional madura e sustentável localiza-se numa boa gestão conjunta do conhecimento. Assim, a boa gestão do conhecimento é potencialmente importante para a geração de novos rents, principalmente nos casos de relacionamentos interorganzacionais que se caracterizam por não terem fins lucrativos.

Assim sendo, para pesquisas futuras, sugere-se uma investigação aprofundada acerca do fluxo de conhecimento durante as fases de compartilhamento de conhecimento propostas por Tonet e Paz (2004). Entretanto, a partir das reflexões realizadas, julga-se importante separar a investigação em dois grandes subconjuntos teóricos, destacando-se a gestão do conhecimento denominado gerencial e a gestão do conhecimento social. Ou seja, investigar quais são as principais semelhanças e diferenças nos conceitos centrais e tecnologias de gestão para esses dois conjuntos.

Os autores da presente pesquisa reconhecem as limitações existentes tanto relativas às escolhas da literatura sobre os assuntos abordados quanto aos critérios metodológicos escolhidos. Estudos de casos refletem parcialmente a realidade daquele momento, com aqueles atores envolvidos e se limitam pelo tempo, espaço e subjetividades inerentes tanto ao pesquisador quanto aos entrevistados.

\section{REFERÊNCIAS}

ACWORTH, E. B. University-industry engagement: The formation of the Knowledge Integration Community (KIC) model at the Cambridge-MIT Institute. Research Policy, v. 37, n. 8, p. 1241-1254, Sep 2008. ISSN 0048-7333. Disponível em: < <Go to ISI >://WOS:000259129300007 >.

ANDRIOF, J.; WADDOCK, S. Unfolding stakeholder engagement. In: ANDRIOF, J.;WADDOCK, S. et al. (Ed.). Unfolding Stakeholder Thinking: Theory, Responsibility and Engagement. Sheffield: Greenleaf Publishing., 2002. p.19-42

APA, M. C. G. D. R. Análise do desenvolvimento do processo de responsabilidade social nas organizações: estudo de caso da empresa sul catarinense mineração, artefatos de cimento, britagem e construção Ltda. Curitiba, PR, Brasil: Anais do Global Forum America Latina. 12008.

ARVANITIS, S.; KUBLI, U.; WOERTER, M. University-industry knowledge and technology transfer in Switzerland: What university scientists think about co-operation with private enterprises. Research Policy, v. 37, n. 10, p. 1865-1883, Dec 2008. ISSN 0048-7333. Disponível em: < <Go to ISI>:// WOS:000261658700018>.

ASTLEY, W. G. TOWARD AN APPRECIATION OF COLLECTIVE STRATEGY. Academy of Management Review, v. 9, n. 3, p. 526-535, 1984 1984. ISSN 0363-7425. Disponível em: < <Go to ISI>:// WOS:A1984TC07900013 >.

AUSTIN, J. The Collaboration Challenge: How Nonprofits and Businesses Succeed through Strategic Alliances. San Fracisco: Jossey-Bass 2000.

BARDIN, L. Análise de conteúdo. Lisboa: Setenta 1977.

BARRINGER, B. R.; HARRISON, J. S. Walking a tightrope: Creating value through interorganizational relationships. Journal of Management, v. 26, n. 3, p. 367-403, 2000 2000. ISSN 0149-2063. Disponível em: < <Go to ISI >://WOS:000088751800001 >. 
BALESTRIN, A. A dinâmica da complementaridade de conhecimentos no contexto das redes interorganizacionais (Tese de Doutorado). Porto Alegre, RS, Brasil: Universidade Federal do Rio Grande do Sul.

BEKKERS, R.; B, F. I. M. Analysing knowledge transfer channels between universities and industry: To what degree do sectors also matter? Research Policy: 1837-1853 p. 2008.

CAPLAN, K. Creating Space for Innovation: Understanding enablers for multi-sector partnerships. Partnership Matters - Special Edition for The Partnering Event. 4: 11-14 p. 2006.

CHILD, J. Organization design and performance: contingency theory and beyond. Birmingham: Aston University 1976.

CHOO, C. W. A organização do conhecimento: como as organizações usam a informação para criar significado, construir conhecimento e tomar decisões. SENAC: São Paulo: 2006.

CUNHA, C. R.; C., M. R. A confiança nos relacionamentos interorganizacionais: o Campo da biotecnologia em análise. RAE Eletrônica, 2006.

COHEN, W. M.; LEVINTHAL, D. A. ABSORPTIVE-CAPACITY - A NEW PERSPECTIVE ON LEARNING AND INNOVATION. Administrative Science Quarterly, v. 35, n. 1, p. 128-152, Mar 1990. ISSN 0001-8392. Disponível em: < <Go to ISI>://WOS:A1990CV83400006 >.

COOK, S. D. N.; BROWN, J. S. Bridging epistemologies: The generative dance between organizational knowledge and organizational knowing. Organization Science, v. 10, n. 4, p. 381-400, Jul-Aug 1999. ISSN 1047-7039. Disponível em: < <Go to ISI >://WOS:000083714300001 >

COURTNEY, J. F. Decision making and knowledge management in inquiring organizations: toward a new decision-making paradigm for DSS. Decision Support Systems, v. 31, n. 1, p. 17-38, May 2001. ISSN 0167-9236. Disponível em: < <Go to ISI>://WOS:000167836400003 >.

D'ESTE, P.; PATEL, P. University-industry linkages in the UK: What are the factors underlying the variety of interactions with industry? Research Policy: 1295-1313 2007.

DANTAS, E. The development of knowledge networks in latercomer innovation systems: the case of Petrobras in the Brazilian offshore oil industry (doctoral dissertation). . University of Sussex, Brighton, England Science and Technology Policy Studies 2006.

DONALDSON, T.; PRESTON, L. E. THE STAKEHOLDER THEORY OF THE CORPORATION - CONCEPTS, EVIDENCE, AND IMPLICATIONS. Academy of Management Review, v. 20, n. 1, p. 65-91, Jan 1995. ISSN 0363-7425. Disponível em: < <Go to ISI > ://WOS:A1995QF07900008 >.

DORADO, S.; GILES, D. E., JR.; WELCH, T. C. Delegation of Coordination and Outcomes in Cross-Sector Partnerships The Case of Service Learning Partnerships. Nonprofit and Voluntary Sector Quarterly, v. 38, n. 3, p. 368-391, Jun 2009. ISSN 0899-7640. Disponível em: < <Go to ISI >://WOS:000265959400001 >.

DYER, J. H.; SINGH, H. The relational view: Cooperative strategy and sources of interorganizational competitive advantage. Academy of Management Review, v. 23, n. 4, p. 660-679, Oct 1998. ISSN 0363-7425. Disponível em: < <Go to ISI>://WOS:000076489300007 >.

EISENHARDT, K. M. BUILDING THEORIES FROM CASE-STUDY RESEARCH. Academy of Management Review, v. 14, n. 4, p. 532-550, Oct 1989. ISSN 0363-7425. Disponível em: < <Go to ISI>:// WOS:A1989AV14400005 >.

FONTANA, R.; GEUNA, A.; MATT, M. Factors affecting university-industry R\&D projects: The importance of searching, screening and signalling. Research Policy, v. 35, n. 2, p. 309-323, Mar 2006. ISSN 00487333. Disponível em: < <Go to ISI >://WOS:000236490600008 >.

FREEMAN, R. E. Stakeholder Thinking: the State of the Art. Helsinki In: NÄSI, J. (Org.) Understanding Stakeholder Thinking (p. 35-46). LSR Publications 1995.

GOMES, R.; LIDDLE, J.; GOMES, L. D. M. Cross-Cultural Analysis of Stakeholder Identification in Municipal Districts. Public Management Review, v. 12, n. 1, p. 53-75, 2010. ISSN 1471-9037. Disponível em: $<<$ Go to ISI >://WOS:000277674900004 >.

GOPALAKRISHNAN, S.; SANTORO, M. D. Distinguishing between knowledge transfer and technology transfer activities: The role of key organizational factors. Ieee Transactions on Engineering Management, v. 51, n. 1, p. 57-69, Feb 2004. ISSN 0018-9391. Disponível em: < <Go to ISI>://WOS:000188950500006 >. 
GREENING, D. W. ; GRAY, B. TESTING A MODEL OF ORGANIZATIONAL RESPONSE TO SOCIAL AND POLITICAL ISSUES. Academy of Management Journal, v. 37, n. 3, p. 467-498, Jun 1994. ISSN 0001-4273. Disponível em: < <Go to ISI>://WOS:A1994NN57600002 >.

HOLMES, S.; MOIR, L. Developing a conceptual framework to identify corporate innovations through engagement with non-profit stakeholders. Corporate Governance: 414-422 p. 2007.

LAMBERT, D. M.; EMMELHAINZ, M. A.; GARDNER, T. Developing and implementing supply chain partnerships: The International_Journal of Logistics Management: 1-17 p. 1996.

LUCCHI, M. O trabalho em equipes multidisciplinares: estudo sobre a mobilização do conhecimento a e aprendizagem numa organização de produtos complexos (Dissertação de mestrado). Vitória, ES, Brasil 2009: Universidade Federal do Espírito Santo. 2009.

LYRA, M. G. Análise de Stakeholder e processo de sustentabilidade empresarial: o caso da empresa Alfa In: I. E. D. E. E. R. S. J. V. Econômico. Responsabilidade Social das Empresas - A contribuição das universidades. São Paulo: Petrópolis 6: 213-255 p. 2008.

NONAKA, I.; TAKEUCHI, H. Criação de conhecimento na empresa. Rio de Janeiro: Campus, 1997.

ORRÙ, M., BIGGART, N. W., HAMILTON, G. G. The new institutionalism in organizational analysis. Chicago: The University of Chicago Press. 1991.

PETROBRÁS. Relatório Social e Ambiental da Petrobrás. 2006 Disponível em: <www.petrobras.com.br>

PFEFFER, J.; SALANCIK, G. R. The external control of organizations: a resource dependence perspective. New York: Harper and How. 1978.

POSTAL, A. M.; M., F. A. Diálogo com públicos estratégicos. Curitiba, PR, Brasil: Anais do Global Forum América Latina, 2008.

POWELL, W. W.; DIMAGGIO, P. J. The New Institutionalism in Organizational Analysis. Chicago and London: The University of Chicago Press: 01-38 p. 1991.

SEITANIDI, M. M. The Future Challenges of Cross Sector Interactions: Interactions between NonProfit Organisations and Businesses. Uxbridge: Brunel University: 15 p. 2007.

STRAUSS, A.; CORBIN, J. Basics of Qualitative Research - Grounded Theory, procedures and techniques. 1990. Newbury Park 270. p.

TONET, H. C.; PAZ, M. D. G. T. D. Um Modelo para o Compartilhamento de Conhecimento no Trabalho. Revista de Administração Contemporânea. 75-94 p. 2004.

VOLPON, C. T. Alinhamento estratégico da responsabilidade socioambiental corporativa em empresas que atuam em redes de relacionamento: resultados de pesquisa na Petrobras. Rio de Janeiro. RJ, Brasil.: Pontifícia Universidade Católica Rio de Janeiro.: 171 p. 2006.

WALKER, C. Philanthropy: social capital or strategic alliance? The involvement of senior UK business executives with the voluntary sector and implications for corporate fundraising.: International Journal of Nonprofit and Voluntary Sector Marketing: 219-239 p. 2002.

JANOWICZ-PANJAITAN, M.; NOORDERHAVEN, N. G. Formal and informal interorganizational learning within strategic alliances. Research Policy, v. 37, n. 8, p. 1337-1355, Sep 2008. ISSN 0048-7333. Disponível em: < <Go to ISI >://WOS:000259129300014 >

KANTER, R. M. From spare change to real change - The social sector as beta site for business innovation. Harvard Business Review, v. 77, n. 3, p. 122-+, May-Jun 1999. ISSN 0017-8012. Disponível em: < <Go to ISI >://WOS:000079984100014 >.

OLIVER, C. DETERMINANTS OF INTERORGANIZATIONAL RELATIONSHIPS - INTEGRATION AND FUTUREDIRECTIONS. Academy of Management Review, v. 15, n. 2, p. 241-265, Apr 1990. ISSN 0363-7425. Disponível em: < <Go to ISI>://WOS:A1990CZ06700003 >

OLIVER, C. STRATEGIC RESPONSES TO INSTITUTIONAL PROCESSES. Academy of Management Review, v. 16, n. 1, p. 145-179, Jan 1991. ISSN 0363-7425. Disponível em: < <Go to ISI>:// WOS:A1991ER54400007 > 
PERKMANN, M.; WALSH, K. University-industry relationships and open innovation: Towards a research agenda. International Journal of Management Reviews, v. 9, n. 4, p. 259-280, Dec 2007. ISSN 1460-8545. Disponível em: < <Go to ISI>://WOS:000251191300001 >.

POWELL, W. W. Learning from collaboration: Knowledge and networks in the biotechnology and pharmaceutical industries. California Management Review, v. 40, n. 3, p. 228-+, Spr 1998. ISSN 0008-1256. Disponível em: < <Go to ISI >://WOS:000073954500013 >.

SCOTT, W. R. THE ADOLESCENCE OF INSTITUTIONAL THEORY. Administrative Science Quarterly, v. 32, n. 4, p. 493-511, Dec 1987. ISSN 0001-8392. Disponível em: < <Go to ISI>://WOS:A1987N352800001 $>$.

SELSKY, J. W.; PARKER, B. Cross-sector partnerships to address social issues: Challenges to theory and practice. Journal of Management, v. 31, n. 6, p. 849-873, Dec 2005. ISSN 0149-2063. Disponível em: <Go to ISI > ://WOS:000233338000003 >.

WIERZBICKI, A. P. Modelling as a way of organising knowledge. European Journal of Operational Research, v. 176, n. 1, p. 610-635, Jan 1 2007. ISSN 0377-2217. Disponível em: < <Go to ISI>:// WOS:000241709600042 >.

YIN, R. K. Estudo de caso - planejamento e métodos. 2. ed. <st1: city w:st="on"><st1:place w:st="on"> Porto Alegre Bookman: Bookman: 205 p. 2001.

YUSUF, S. Intermediating knowledge exchange between universities and businesses. Research Policy, v. 37, n. 8, p. 1167-1174, Sep 2008. ISSN 0048-7333. Disponível em: < <Go to ISI>:// WOS:000259129300002 >. 